\title{
Shakedown of discrete systems involving plasticity and friction
}

Anders Klarbring, J . R. Barber, A. Spagnoli and M. Terzano

The self-archived version of this journal article is available at Linköping University Electronic Press:

http:/ / urn.kb.se/ resolve?urn=urn:nbn:se:liu:diva- 137370

N.B.: When citing this work, cite the original publication.

Klarbring, A., Barber, J . R., Spagnoli, A., Terzano, M., (2017), Shakedown of discrete systems involving plasticity and friction, European journal of mechanics. A, Solids, 64, 160-164.

https:// dx.doi.org/ 10.1016/j.euromechsol.2017.02.006

Original publication available at:

https:// dx.doi.org/ 10.1016/j.euromechsol.2017.02.006

Copyright: Elsevier

http:// www.elsevier.com/

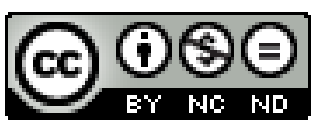




\title{
Shakedown of discrete systems involving plasticity and friction
}

\author{
A. Klarbring ${ }^{\mathrm{a}}$, J. R. Barber ${ }^{\mathrm{b}}$, A. Spagnolic,*, M. Terzano ${ }^{\mathrm{c}}$ \\ ${ }^{a}$ Department of Mechanical Engineering, Linköping University, S-581 83 Linköping, \\ Sweden \\ ${ }^{b}$ Department of Mechanical Engineering, University of Michigan, Ann Arbor, MI \\ 48109-2125, USA \\ ${ }^{c}$ Department of Engineering and Architecture, University of Parma, Viale Usberti \\ 181/A, 43124 Parma, Italy
}

\begin{abstract}
In associated plasticity, systems subjected to cyclic loading are sometimes predicted to shake down, meaning that, after some dissipative cycles, the response goes back to a purely elastic state, where no plastic flow occurs. Frictional systems show a similar behaviour, in the sense that frictional slips due to the external loads may cease after some cycles. It has been proved that, for complete contacts with elastic behaviour and Coulomb friction, Melan's theorem gives a sufficient condition for the system to shake down, if and only if there is no normal-tangential coupling at the interfaces. In this paper, the case of discrete systems combining elastic-plastic behaviour and Coulomb friction is considered. In particular, it is proved that Melan's theorem still holds for contact-wise uncoupled systems, i.e., the existence of a residual state, comprised of frictional slips and plastic strains, is a sufficient condition for the system to shake down.
\end{abstract}

Keywords: Contact problems; Limit analysis; Shakedown; Melan's theorem; Coulomb friction

\footnotetext{
${ }^{*}$ Corresponding author.

Email address: spagnoli@unipr.it (A. Spagnoli)
} 


\section{Introduction}

Systems with an elastic-plastic behaviour may show different responses under cyclic loading, depending on the magnitude of the applied loads. Sometimes, they might develop plastic deformations at the early stages, which cease after some loading cycles, in a way that the response is no longer dissipative, and each subsequent cycle is purely elastic. This event commonly goes under the name of shakedown. The knowledge of the limit load under which shakedown occurs is an issue of fundamental importance in mechanics and limit analysis provides the two main theorems which are commonly used to calculate this limit in elastic-perfectly plastic solids. In particular, the lower-bound theorem, also known as Melan's theorem, follows a static approach, based on the existence of a residual self-equilibrated stress state, which provides both a necessary and sufficient condition for the shakedown (Melan, 1936).

When systems with parts in mutual contact are subjected to cyclic loading, frictional slips may occur along the interfaces, leading to the well-known phenomenon of fretting fatigue. However, if the interfaces are conforming, that is, the contact is complete, it is possible that the system, after some loading cycles, reaches a state where slip is inhibited (Churchman and Hills, 2006). It is evident that this behaviour shares many similarities with the response in elastic-plastic solids, if frictional slip is thought of as being analogous to plastic strain (see, for instance, Carpinteri and Scavia, 1993; Churchman et al., 2006). The situation of adhered contact interfaces, after some cycles where slip has occurred, exhibit the same behaviour as elastic-plastic systems that have reverted to an elastic response after plastic flow has occurred.

These remarks have prompted the question as to whether a theorem analogous to Melan's could be developed for frictional contacts. Such a theorem shall establish a sufficient condition for shakedown on the existence of a residual displacement distribution that would prevent slip at all times during load cycles. Regretfully, the non-associative nature of the friction law prevents the limit analysis theorems to be applied directly (Drucker, 1954). In the field of plasticity, shakedown analysis has been extended beyond the limits of standard materials in order to include friction type material laws. This prompted scholars to focus on the implications of a non-associated flow rule (see, for instance, Maier, 1969; Pycko and Maier, 1995), and a different formulation

of the lower and upper bound theorems for the shakedown limit load was 
obtained. A new approach in shakedown analysis for a general non-standard material law has been recently proposed by Bousshine et al. (2003), grounded on the so-called bi-potential theory. Shakedown of systems with boundary contact conditions has also been considered, for instance, in Telega (1995) and Polizzotto (1997).

Shakedown of frictional systems with elastic behaviour has been investigated recently by Ahn et al. (2008) and Flicek et al. (2015). An analogous of Melan's theorem has been proved for discrete contact problems (Klarbring et al., 2007) and for continuum problems (Barber et al., 2008), thanks to the following fundamental remark: when normal reactions on the slip surface can can be regarded as defined from equilibrium alone, the normality rule applies on the tangential plane and thus the flow rule is associated (Michalowski and Mroz, 1978). Therefore, introducing the hyphotesis of uncoupled contact, we are assuming the distribution of normal contact reactions not influenced by relative tangential contact displacements, thus making the problem associated.

To the authors best knowledge, the issue of the shakedown with respect to plasticity and friction has attracted no attention. In the present paper, the case of a discrete system with elastic-plastic behaviour and frictional interfaces is considered, with the aim of proving that an analogous theorem to Melan's still holds if the contact is uncoupled.

\section{Formulation}

We consider a system which is discretized, e.g. by means of the finite element method, such that we can define a vector of nodal displacements $\boldsymbol{u}$ and a vector of given external nodal forces $\boldsymbol{F}$. Let $\varepsilon$ be the vector which collects strains $\left(\varepsilon_{i j}\right)_{k}$ at integration points of the discretized system. These strains, under the assumption of small displacements, are connected to the nodal displacements by means of a strain-displacement matrix $\boldsymbol{B}$. Then we can write

$$
\varepsilon=\boldsymbol{B u}
$$

If we assume an elastic-plastic material behaviour, strains are decomposed into an elastic and a plastic part, and a vector $\boldsymbol{\sigma}$ of stresses at the integration points is defined such that

$$
\begin{gathered}
\varepsilon=e+p \\
\sigma=E e
\end{gathered}
$$


where $\boldsymbol{E}$ is the elasticity matrix.

Let us now assume that the system features complete contact interfaces. We can collect the contact displacements, which are relative displacements in case of two-body contact, in a vector $\boldsymbol{w}$, whose components are expressed in a local coordinate system, aligned in normal and tangential contact directions. Then we introduce a transformation matrix $\boldsymbol{C}$ such that

$$
\boldsymbol{w}=\boldsymbol{C u}
$$

At contact nodes we can also collect the contact reaction forces in a vector $\boldsymbol{r}$, where $\boldsymbol{r}=\boldsymbol{\kappa} \boldsymbol{w}$, with $\boldsymbol{\kappa}$ being the contact stiffness matrix. This matrix is obtained from the global stiffness matrix $\boldsymbol{K}$ using the transformation matrix $\boldsymbol{C}$ as

$$
\boldsymbol{\kappa}^{-1}=\boldsymbol{C} \boldsymbol{K}^{-1} \boldsymbol{C}^{T}
$$

The global stiffness matrix is obtained as

$$
\boldsymbol{K}=\boldsymbol{B}^{T} \boldsymbol{E D}
$$

where $\boldsymbol{D}=\boldsymbol{V} \boldsymbol{B}$ with $\boldsymbol{V}$ being a diagonal matrix containing the volume associated to each integration point of finite elements.

The global equilibrium of the system is described by the equation

$$
\boldsymbol{F}+\boldsymbol{C}^{T} \boldsymbol{r}=\boldsymbol{D}^{T} \boldsymbol{\sigma}
$$

\subsection{The yield condition}

To state the yield condition of the elastic-plastic system, we need to introduce a convex yield function and an associated flow rule. On an individual integration point $k$ we can write

$$
f_{k}\left(\boldsymbol{\sigma}_{k}\right) \leq 0
$$

where $\boldsymbol{\sigma}_{k}$ is the vector of total stresses on the $k^{t h}$ integration point.

The associated nature of the flow rule ensures that Drucker's stability postulate applies and the time derivative of the plastic strain $\dot{\boldsymbol{p}}_{k}$ is governed by

$$
\left(\boldsymbol{\sigma}_{k}-\boldsymbol{\sigma}_{k}^{*}\right)^{T} \dot{\boldsymbol{p}}_{k} \geq 0 \quad f_{k}\left(\boldsymbol{\sigma}_{k}^{*}\right)<0
$$

where $\boldsymbol{\sigma}_{k}^{*}$ is any stress vector at the $k^{t h}$ integration point corresponding to a state inside the elastic domain. 


\subsection{The friction law}

To state the conditions of the friction law, let us consider the separation interface of two bodies, $A$ and $B$. We define with $\boldsymbol{u}^{A}$ and $\boldsymbol{u}^{B}$ the displacement vectors and denote with $\boldsymbol{n}^{A}$ and $\boldsymbol{n}^{B}$ the inward unit vectors normal to the interfaces. We may express the normal and tangential components of contact relative displacements as

$$
w_{n}=\boldsymbol{u}^{A} \cdot \boldsymbol{n}^{A}+\boldsymbol{u}^{B} \cdot \boldsymbol{n}^{B}, \quad \boldsymbol{w}_{\boldsymbol{t}}=\boldsymbol{u}_{\boldsymbol{t}}^{A}-\boldsymbol{u}_{\boldsymbol{t}}^{B}
$$

where a central dot indicates the standard scalar product of geometric vectors and $\boldsymbol{u}_{\boldsymbol{t}}^{J}=\boldsymbol{u}^{J}-\left(\boldsymbol{u}^{J} \cdot \boldsymbol{n}^{J}\right) \boldsymbol{n}^{J},(J=A, B)$. According to this notation, a normal relative displacement is positive when it opens a gap, therefore the non-penetration condition will be written $w_{n} \geq 0$. In case one of the bodies is rigid, we can assume $\boldsymbol{u}^{B}=0$.

On each node $i$, we may write $\boldsymbol{n}_{i}^{A}=-\boldsymbol{n}_{i}^{B}=\boldsymbol{n}_{i}$ and therefore we have

$$
\boldsymbol{w}_{i}=\boldsymbol{w}_{i t}+w_{i n} \boldsymbol{n}_{i}, \quad \boldsymbol{w}_{i t} \cdot \boldsymbol{n}_{i}=0
$$

Similarly, we decompose the contact reaction forces in normal and tangential components as

$$
\boldsymbol{r}_{i}=\boldsymbol{r}_{i t}+r_{i n} \boldsymbol{n}_{i}, \quad \boldsymbol{r}_{i t} \cdot \boldsymbol{n}_{i}=0
$$

The following equations set the conditions of Coulomb friction law

$$
\begin{aligned}
\left|\boldsymbol{r}_{i t}\right| & \leq \mu_{i} r_{i n} \\
0<\left|\boldsymbol{r}_{i t}\right| & =\mu_{i} r_{i n} \Longrightarrow \dot{\boldsymbol{w}}_{i t}=-\lambda_{i} \boldsymbol{r}_{i t}, \lambda_{i} \geq 0 \\
\left|\boldsymbol{r}_{i t}\right| & <\mu_{i} r_{i n} \Longrightarrow \dot{\boldsymbol{w}}_{i t}=\mathbf{0}
\end{aligned}
$$

where $\mu_{i}$ is the coefficient of friction at node $i$ and a superposed dot denotes time derivative. This law specifies when and how slip takes place: slip is zero when contact forces belong to an admissible set, the so called Coulomb cone of friction, and it otherwise opposes the tangential force. Moreover, we are considering a system with known contact interfaces, therefore $w_{\text {in }}=0$. In such a case, the hyphothesis of normal-tangential uncoupling has an evident consequence on the contact stress state. Indeed, we say that the contact is uncoupled if, for a displacement $\boldsymbol{w}$ with $w_{\text {in }}=0$ for all $i$, it holds that $\boldsymbol{r}=\boldsymbol{\kappa} \boldsymbol{w}$ is such that $r_{i n}=0$. 


\subsection{Residual state}

Let us define the residual state of the system, denoted by index $R$, that is, when the external loads are removed, $\boldsymbol{F}=\mathbf{0}$, keeping $\boldsymbol{p}$ and $\boldsymbol{w}$ fixed. The response of the system is described by means of the following equations

$$
\begin{gathered}
\boldsymbol{\varepsilon}_{R}=\boldsymbol{e}_{R}+\boldsymbol{p} \\
\boldsymbol{\varepsilon}_{R}=\boldsymbol{B} \boldsymbol{u}_{R} \\
\boldsymbol{\sigma}_{R}=\boldsymbol{E} \boldsymbol{e}_{R} \\
\boldsymbol{w}=\boldsymbol{C} \boldsymbol{u}_{R} \\
\boldsymbol{C}^{T} \boldsymbol{r}_{R}=\boldsymbol{D}^{T} \boldsymbol{\sigma}_{R}
\end{gathered}
$$

where $\boldsymbol{u}_{R}$ is the displacement vector and $\boldsymbol{r}_{R}$ and $\boldsymbol{\sigma}_{R}$ are the contact reactions and residual stress vectors. Equation (18) describes the global equilibrium in this state. We can note that compatibility requires the strain vector in (14) to be the sum of an elastic component and a plastic component.

Combining equations (14) - (18) with (6) and (5), we are able to express the residual vectors as a function of $\boldsymbol{w}$ and $\boldsymbol{p}$

$$
\begin{gathered}
\boldsymbol{\sigma}_{R}=\boldsymbol{E}(\boldsymbol{B} \boldsymbol{w}-\boldsymbol{p}) \\
\boldsymbol{r}_{R}=\boldsymbol{\kappa}\left(\boldsymbol{w}-\boldsymbol{C} \boldsymbol{K}^{-1} \boldsymbol{D}^{T} \boldsymbol{E} \boldsymbol{p}\right)
\end{gathered}
$$

Reintroducing the external loads, we obtain an elastic process, denoted by index $E$, which can be simply added to the residual state, so that the response of the system is described by means of the following equations

$$
\begin{gathered}
\boldsymbol{\varepsilon}=\boldsymbol{e}_{E}+\boldsymbol{e}_{R}+\boldsymbol{p} \\
\boldsymbol{e}_{E}=\boldsymbol{B} \boldsymbol{u}_{E} \\
\boldsymbol{\sigma}_{E}=\boldsymbol{E} \boldsymbol{e}_{E} \\
\mathbf{0}=\boldsymbol{C} \boldsymbol{u}_{E} \\
\boldsymbol{F}+\boldsymbol{C}^{T} \boldsymbol{r}_{E}=\boldsymbol{D}^{T} \boldsymbol{\sigma}_{E} \\
\boldsymbol{u}=\boldsymbol{u}_{R}+\boldsymbol{u}_{E} \\
\boldsymbol{r}=\boldsymbol{r}_{R}+\boldsymbol{r}_{E},
\end{gathered}
$$


The elastic part of the strain vector is $\boldsymbol{e}=\boldsymbol{e}_{R}+\boldsymbol{e}_{E}$, so by (3), (16) and (23) we have

$$
\boldsymbol{\sigma}=\boldsymbol{\sigma}_{R}+\boldsymbol{\sigma}_{E}
$$

Notice that the residual state of equations (14-20) is not required to satisfy the frictional inequalities, since the external loads are never actually removed. Only the combined state of equations (21-28) is required to satisfy these conditions.

\section{Shakedown}

The elastic-plastic system with frictional contact interfaces under consideration is submitted to cyclic loading with period $T$, such that an initial transient stage is followed by a cyclic stage. We define a steady state by assuming that, at a certain time instant $t$, the stress state of the system becomes a periodic function with period equal to that of the external loading, that is, $\boldsymbol{\sigma}(t)=\boldsymbol{\sigma}(t+T)$ and $\boldsymbol{r}(t)=\boldsymbol{r}(t+T)$. In order to investigate the response of such a system and outline the conditions for the shakedown, we initially analyze separately the shakedown with respect to plasticity alone and the shakedown of the frictional contact. We also note that the first case originates if we consider the contact interface to be permanently welded, while the latter is made possible by assuming perfectly elastic response of the material.

\subsection{Shakedown in plasticity}

In an elastic-plastic system, the steady state cycle might be characterized by plastic strain, whose increment in the load cycle $T$ is defined as

$$
\Delta \boldsymbol{p}=\int_{t}^{t+T} \dot{\boldsymbol{p}} d t
$$

Three fundamental situations can be distinguished, as shown in figure 1(a):

(i) $\Delta \boldsymbol{p} \neq \mathbf{0}$. Ratcheting. A progressive increment of plastic strain occurs at each steady state cycle leading to an incremental collapse;

(ii) $\dot{\boldsymbol{p}} \neq \mathbf{0}, \Delta \boldsymbol{p}=\mathbf{0}$. Alternating plasticity. Plastic strain of opposite sign sums up so that the plastic increment is null but energy is dissipated at each cycle; 

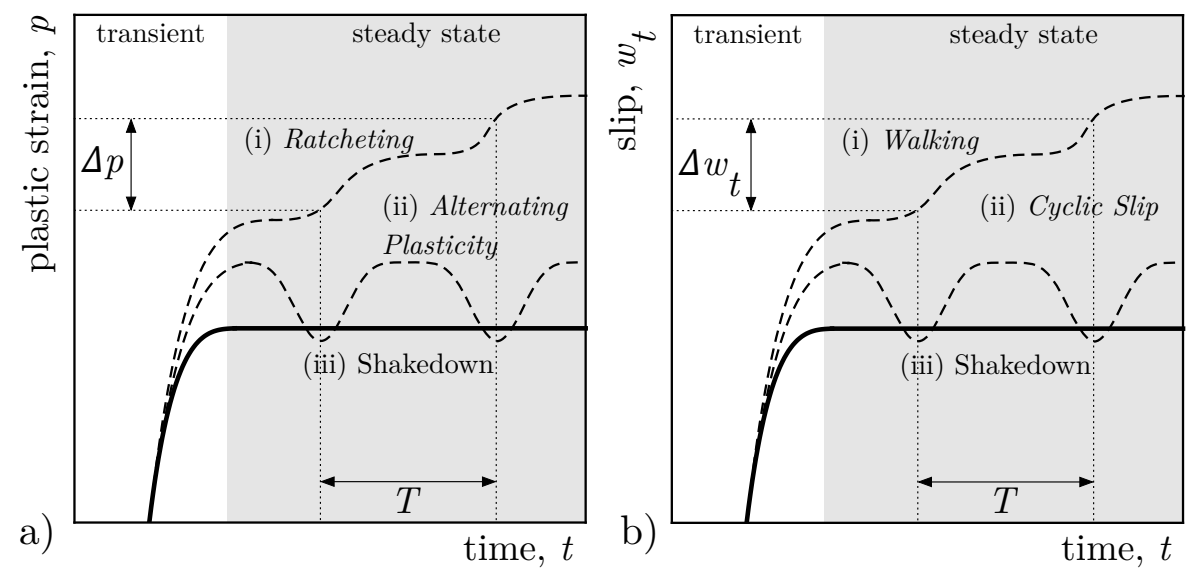

Figure 1. System response to cyclic loading (schematic). (a) Response in an elastic-plastic system, (b) response of frictional contact.

(iii) $\dot{\boldsymbol{p}}=\mathbf{0}$. Shakedown. Plastic strain ceases after the initial transient stage and no energy is dissipated in the steady state.

According to (iii), the system will have reached a state of shakedown if, at any time after the initial transient stage, $\dot{\boldsymbol{p}}=\mathbf{0}$, i.e., no plastic flow occurs, in any integration point of the discretized system. Using the theorems of limit analysis, specifically Melan's static theorem, we can investigate this occurrence.

Let us consider the elastic response to external loading described by the vector of time varying stresses $\boldsymbol{\sigma}_{E}(t)$, which satisfies the equation of equilibrium. According to Melan's theorem, a necessary and sufficient condition for adaptation is the existence of a residual time-independent stress state $\tilde{\boldsymbol{\sigma}}_{R}$ such that, for all times, $\tilde{\boldsymbol{\sigma}}=\tilde{\boldsymbol{\sigma}}_{R}+\boldsymbol{\sigma}_{E}$ satisfies the yield condition $f_{k}\left(\tilde{\boldsymbol{\sigma}}_{k}\right) \leq 0$.

An evident advantage of Melan's theorem is that the actual time-dependent elastic-plastic stress in the body does not have to be determined. The elastic solution is superimposed on a self-equilibrated stress distribution which may differ from the actual distribution due to the particular initial load path, as long as the resulting stress state is admissible with respect to yielding. 


\subsection{Shakedown in frictional contacts}

In a system with a complete contact interface, the steady state cycle might be characterized by frictional slips and therefore, in the load cycle $T$, the cumulated tangential displacement is defined as

$$
\Delta \boldsymbol{w}_{\boldsymbol{t}}=\int_{t}^{t+T} \dot{\boldsymbol{w}}_{t} d t
$$

Three fundamental situations can be distinguished, as shown in figure 1(b):

(i) $\Delta \boldsymbol{w}_{\boldsymbol{t}} \neq \mathbf{0}$. Walking. Each loading cycle produces slips, therefore we will have a net cumulated displacement leading to a rigid body motion (Mugadu et al., 2004);

(ii) $\dot{\boldsymbol{w}}_{\boldsymbol{t}} \neq \mathbf{0}, \Delta \boldsymbol{w}_{\boldsymbol{t}}=\mathbf{0}$. Cyclic slip. Slips of opposite sign occur but the cumulated displacement is zero;

(iii) $\dot{\boldsymbol{w}}_{\boldsymbol{t}}=\mathbf{0}$. Shakedown. Slips cease after the initial transient stage and no energy is dissipated in the steady state.

According to (iii), the system will have reached a state of shakedown if, at any time after the initial transient stage, $\dot{\boldsymbol{w}}_{\boldsymbol{t}}=\mathbf{0}$, i.e., no frictional slip occurs and all contact nodes remain in a state of stick. Following a static formulation analogous to Melan's theorem, a necessary condition for frictional shakedown has been established (Klarbring et al., 2007). It has been proved that this condition is also sufficient if and only if the contact is uncoupled.

Let us consider the contact response to external loading described by the vector of time varying contact reaction forces $\boldsymbol{r}_{E}$. According to the frictional shakedown theorem, a necessary and sufficient condition for adaptation is the existence of a time-independent vector $\tilde{\boldsymbol{w}}$ such that, for all times, $\tilde{\boldsymbol{r}}=\boldsymbol{r}_{R}+\boldsymbol{r}_{E}$ satisfies Coulomb slip condition $\left|\tilde{\boldsymbol{r}}_{i t}\right| \leq \mu_{i} \tilde{r}_{i n}$.

\subsection{Shakedown with plasticity and friction}

We now consider the shakedown combining both friction and plasticity. Therefore, in a steady state cycle, the two following conditions shall be satisfied

$$
\dot{p}=\mathbf{0}, \quad \dot{\boldsymbol{w}}=\mathbf{0}
$$


According to the previous statement, an elastic-plastic frictional system will have reached a state of shakedown if, at any time after the initial transient stage, (i) no plastic flow and (ii) no frictional slip occurs. In this situation, two limit conditions must be satisfied, namely the yield condition in (8) and the limit condition defined by Coulomb friction law in (13a). Evidently, these conditions define two different limit surfaces, as the first refers to stresses in the integration points of the system, while the latter is expressed as a limit condition in terms of contact reaction forces on the nodes along the interface.

Moreover, we also note that possibly only one of these conditions will be satisfied as an equality, while the other will be simply verified as a strict inequality. The occurrence of one rather than the other limit condition clearly depends on the coefficient of friction, on the yield stress of the material and

on the features of the system, in terms of contact geometry and external load distribution.

\section{Shakedown Theorem}

Following the usual formulation of the static shakedown theorem, a necessary condition for shakedown in an elastic-plastic frictional system is the existence a residual time-independent state $\left(\tilde{\boldsymbol{\sigma}}_{R}, \tilde{\boldsymbol{r}}_{R}\right)$ such that, for all times, $\tilde{\boldsymbol{\sigma}}=\tilde{\boldsymbol{\sigma}}_{R}+\boldsymbol{\sigma}_{E}$ satisfies

$$
f_{k}\left(\tilde{\boldsymbol{\sigma}}_{k}\right) \leq 0
$$

and $\tilde{\boldsymbol{r}}=\tilde{\boldsymbol{r}}_{R}+\boldsymbol{r}_{E}$ satisfies

$$
\left|\tilde{\boldsymbol{r}}_{i t}\right| \leq \mu_{i} \tilde{r}_{i n}
$$

where the residual state is defined similarly to (19) and (20). We refer to it as a safe residual state if the strict inequalities in the previous conditions are enforced.

Our purpose is to define a theorem which provides a sufficient condition for shakedown in a discrete system, using the notion of no elastic coupling between normal and tangential contact directions. Before giving its formulation, we provide a modified definition of shakedown by introducing the norm

$$
A=\frac{1}{2}\left(\boldsymbol{\sigma}_{R}-\tilde{\boldsymbol{\sigma}}_{R}\right)^{T} \boldsymbol{E}^{-1}\left(\boldsymbol{\sigma}_{R}-\tilde{\boldsymbol{\sigma}}_{R}\right) \geq 0
$$

which is a measure of the difference between the real residual state $\boldsymbol{\sigma}_{R}$ and the safe residual state $\tilde{\boldsymbol{\sigma}}_{R}$. 
Theorem. Assume that there exists a safe residual state $\left(\tilde{\boldsymbol{\sigma}}_{R}, \tilde{\boldsymbol{r}}_{R}\right)$ such that $\tilde{\boldsymbol{\sigma}}=\tilde{\boldsymbol{\sigma}}_{R}+\boldsymbol{\sigma}_{E}$ satisfies $f_{k}\left(\tilde{\boldsymbol{\sigma}}_{k}\right)<0$ and $\tilde{\boldsymbol{r}}=\tilde{\boldsymbol{r}}_{R}+\boldsymbol{r}_{E}$ satisfies $\left|\tilde{\boldsymbol{r}}_{i t}\right|<\mu_{i} \tilde{r}_{i n}$. If the system has no elastic normal-tangential coupling, then the real residual state $\left(\boldsymbol{\sigma}_{R}, \boldsymbol{r}_{R}\right)$ will approach $\left(\tilde{\boldsymbol{\sigma}}_{R}, \tilde{\boldsymbol{r}}_{R}\right)$ in the sense that $\dot{A}<0$ whenever $\dot{\boldsymbol{p}} \neq \mathbf{0}$ or $\dot{\boldsymbol{w}} \neq \mathbf{0}$.

Proof. The time derivative of the norm $A$ is:

$$
\dot{A}=\left(\boldsymbol{\sigma}_{R}-\tilde{\boldsymbol{\sigma}}_{R}\right)^{T} \boldsymbol{E}^{-1} \dot{\boldsymbol{\sigma}}_{R}=\left(\boldsymbol{\sigma}_{R}-\tilde{\boldsymbol{\sigma}}_{R}\right)^{T}\left(\dot{\boldsymbol{\varepsilon}}-\dot{\boldsymbol{e}}_{E}-\dot{\boldsymbol{p}}\right)
$$

where we have used (16) and (21). From (1), (22) and (18) we get

$$
\dot{A}=-\left(\boldsymbol{\sigma}_{R}-\tilde{\boldsymbol{\sigma}}_{R}\right)^{T} \dot{\boldsymbol{p}}+\left(\boldsymbol{r}_{R}-\tilde{\boldsymbol{r}}_{R}\right)^{T} \boldsymbol{C}\left(\dot{\boldsymbol{u}}-\dot{\boldsymbol{u}}_{E}\right)
$$

and, finally, using (4) and (24) the result is

$$
\dot{A}=-\left(\boldsymbol{\sigma}_{R}-\tilde{\boldsymbol{\sigma}}_{R}\right)^{T} \dot{\boldsymbol{p}}+\left(\boldsymbol{r}_{R}-\tilde{\boldsymbol{r}}_{R}\right)^{T} \dot{\boldsymbol{w}}
$$

where the first term is non-positive since

$$
\left(\boldsymbol{\sigma}_{R}-\tilde{\boldsymbol{\sigma}}_{R}\right)^{T} \dot{\boldsymbol{p}}=(\boldsymbol{\sigma}-\tilde{\boldsymbol{\sigma}})^{T} \dot{\boldsymbol{p}}=\sum_{k}\left(\boldsymbol{\sigma}_{k}-\tilde{\boldsymbol{\sigma}}_{k}\right)^{T} \dot{\boldsymbol{p}}_{k} \geq 0
$$

from Drucker's principle (9), see figure 2(a). If $\dot{\boldsymbol{p}} \neq \mathbf{0}$, it is also strictly negative since the residual state is safe. Regarding the second term of (31), if there is no elastic normal-tangential coupling, both $\boldsymbol{r}_{i}$ and $\tilde{\boldsymbol{r}}_{i}$ belong to the circle of radius $\mu r_{i n}=\mu \tilde{r}_{i n}$ formed by cutting the friction cone at the constant normal force level $r_{i n}=\tilde{r}_{i n}$. According to (13b), $\dot{\boldsymbol{w}}_{i} \neq \mathbf{0}$ is directed along the inward normal to the circle, therefore $\left(\tilde{\boldsymbol{r}}_{i}-\boldsymbol{r}_{i}\right) \cdot \dot{\boldsymbol{w}}_{i}$ is positive, as shown in figure 2(b). Since

$$
\left(\boldsymbol{r}_{R}-\tilde{\boldsymbol{r}}_{R}\right)^{T} \dot{\boldsymbol{w}}=(\boldsymbol{r}-\tilde{\boldsymbol{r}})^{T} \dot{\boldsymbol{w}}=-\sum_{i}\left(\tilde{\boldsymbol{r}}_{i}-\boldsymbol{r}_{i}\right) \cdot \dot{\boldsymbol{w}}_{i}
$$

it is clear that the second term of (31) is also non-positive and strictly negative if $\dot{\boldsymbol{w}} \neq \mathbf{0}$. It follows that $\dot{A}$ is non-positive, thus proving the theorem. 

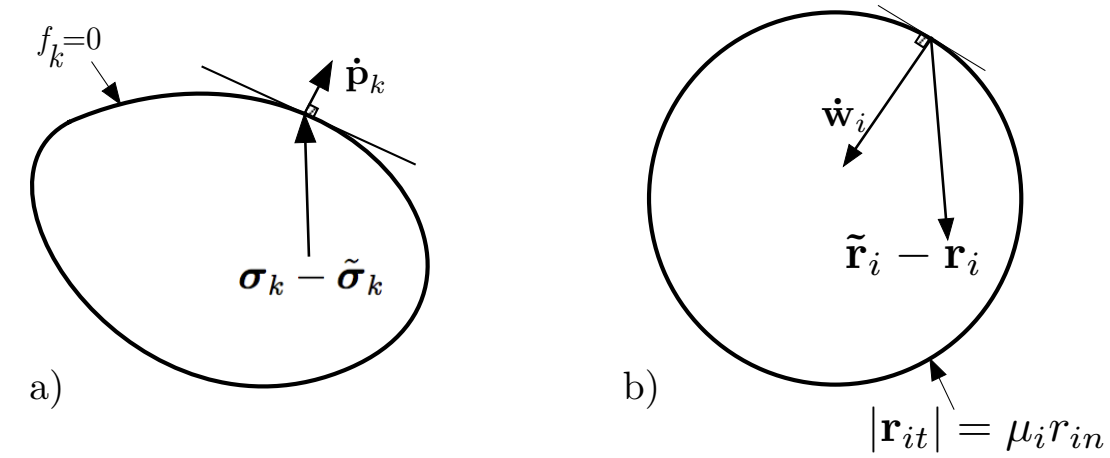

Figure 2. (a) Convex yield function with associated plastic flow. (b) Coulomb's circle, showing vector $\left(\tilde{\boldsymbol{r}}_{i}-\boldsymbol{r}_{i}\right)$ directed inward from the point $\boldsymbol{r}_{i}$ on the boundary.

\section{Conclusions}

The assessment of the condition of shakedown is an issue of great interest in mechanics. In Melan's static theorem, originally formulated in limit analysis of elastic-plastic systems with associated plasticity, the existence of a time-independent residual state gives a sufficient condition for the shakedown. A similar condition applies to elastic systems with frictional interfaces, with the fundamental limitation of normal-tangential uncoupling, in order to respect the associated nature of the flow rule. In this article, we give an exhaustive formulation of the shakedown in a system involving both friction and plasticity and formulate a theorem which provides a sufficient condition for adaptation in the system.

\section{References}

Ahn, Y. J., Bertocchi, E., Barber, J. R., 2008. Shakedown of coupled twodimensional discrete frictional systems. J. Mech. Phys. Solids 56 (12), 3433-3440.

Barber, J. R., Klarbring, A., Ciavarella, M., 2008. Shakedown in frictional contact problems for the continuum. C. R. Mecanique 336 (1-2), 34-41.

Bousshine, L., Chaaba, A., De Saxce, G., 2003. A new approach to shakedown analysis for non-standard elastoplastic material by the bipotential. Int. J. Plasticity 19 (5), 583-598. 
Carpinteri, A., Scavia, C., 1993. Energy dissipation due to frictional shakedown on a closed crack subjected to shear. Meccanica 28 (4), 347-352.

Churchman, C. M., Hills, D. A., 2006. General results for complete contacts subject to oscillatory shear. J. Mech. Phys. Solids 54 (6), 1186-1205.

Churchman, C. M., Korsunsky, A., Hills, D. A., 2006. The Application of Plasticity Principles to Friction. J. Strain Anal. Eng. 41 (4), 323-328.

Drucker, D., 1954. Coulomb friction, plasticity and limit loads. J. Appl. Mech. 21, 71-74.

Flicek, R. C., Hills, D. A., Barber, J. R., Dini, D., 2015. Determination of the shakedown limit for large, discrete frictional systems. Eur. J. Mech. A-Solid. 49, 242-250.

Klarbring, A., Ciavarella, M., Barber, J. R., 2007. Shakedown in elastic contact problems with Coulomb friction. Int. J. Solids Struct. 44 (25-26), 8355-8365.

Maier, G., 1969. Shakedown theory in perfect elastoplasticity with associated and nonassociated flow-laws: A finite element, linear programming approach. Meccanica 4 (3), 250-260.

Melan, E., 1936. Theorie statisch unbestimmter Systeme aus idealplastischem Baustoff. In: Akad. Wiss. Wien Sitzungsber. Vol. 145. pp. 195-218.

Michalowski, R. L., Mroz, Z., 1978. Associated and non-associated sliding rules in contact friction problems. Arch. Mech. 30 (3), 259-276.

Mugadu, A., Sackfield, A., Hills, D., 2004. Analysis of a rocking and walking punch-part I: Initial transient and steady state. J. Appl. Mech. Trans. ASME 71 (2).

Polizzotto, C., 1997. Shakedown of elastic-plastic solids with frictionless unilateral contact boundary conditions. Int. J. Mech. Sci. 39 (7), 819-828.

Pycko, S., Maier, G., 1995. Shakedown theorems for some classes of nonassociative hardening elastic-plastic material models. Int. J. Plasticity 11 (4), 367-395. 
Telega, J. J., 1995. On Shakedown Theorems in the Presence of Signorini Conditions and Friction. In: Mróz, Z., Weichert, D., Dorosz, S. (Eds.), Inelast. Behav. Struct. under Var. Loads. Springer Netherlands, pp. 183201. 